\title{
De la competencia a la cooperación militar en América Latina*
}

La principal constatación que surge de un análisis de las relaciones internacionales contemporáneas, es que la estructura de poder mundial no sólo es cambiante en ciclos Iargos, sino que también muestra transformaciones de significación en ciclos de corto plazo ${ }^{1}$. Este hecho es particularmente cierto y pertinente para el análisis de las relaciones internacionales de América del Sur, en especial para estudiar su inserción en el mundo de los temas estratégicos y comprender sus vinculaciones externas en el campo político-militar.

Desde su emergencia como entes políticos relativamente autónomos, los pafses latinoamericanos han estado bajo la influencia, dominio o hegemonía de alguna potencia extracontinental. Esta relación de subordinación económico-política también se ha manifestado en el campo de las vinculaciones militares, especialmente en materias de incorporación de nuevas doctrinas estratégicas, concepciones tácticas y transferencias de tecnología bélica ${ }^{2}$. Sin embargo, al

*Estas notas son el resultado del seminario final sobre "Paz, Desarme y Desarrollo en América Latina", realizado en Santiago de Chile, los dfas 26 y 27 de mayo de 1986. En esta ocasión se debatió un conjunto de temas, sobre la base de los trabajos presentados en la primera parte de este proyecto. En este scminario final participaron la investigadora señora Pilar Armanet, directora del Instituto de Estudios Internacionales de la Universidad de Chile; el coronel (I) Eduardo Moreno, del Ministerio de Defensa de la República Argentina; el general (r) Edgardo Mercado Jarrín, director del Instituto Peruano de Estudios Geo-estratégicos; el investigador señor Eliezer Rizzo de Oliveira, director del Núcleo de Estudios Estratégicos de la Universidad de Campinas, Brasil; el profesorinvestigador señor Carlos Portales, de FLAcso-Santiago; el profesor señor José Agustfn Silva Michelena, director latinoamericano del proyecto Paz y Transformaciones Globales de la Universidad de las Naciones Unidas; y el profesor señor José Antonio Viera-Gallo, director del Centro de Estudios Sociales (Gesoc), Chile. A todos ellos les debo gran parte de las ideas que aquil se exponen, aun cuando la forma en que ellas han sido interpretadas, es de mi exclusiva responsabilidad.

ISobre los alcances de estas afirmaciones para el caso de la posición internacional de los Estados Unidos, ver: Luis Maira, "Una mirada histórica a los márgenes de hegemonía internacional de Estados Unidos", en: Luis Maira et. al., ¿Una Nueva Era de Hegemonia Norteamericana?, RIAL-GEL, Buenos Aires, 1985, página 15.

${ }_{\text {aAl }}$ respecto, ver: Augusto Varas, "Las relaciones" militares internacionales de 
interior de estos cambiantes ciclos largos de subordinación y dependencia estratégica de América Latina, se han podido observar momentos -o ciclos cortos- de una mayor autonomía relativa frente a la potencia militar rectora del momento. De hecho, la presencia militar alemana y francesa en América del Sur se materializó durante el período de hegemonía británica en la región. De igual forma, el dominio e influencia militar-estratégico de los Estados Unidos en el último período de postguerra, coexistió con una influencia permanente de Inglaterra en materias navales.

Estos ciclos cortos de relativa independencia o permisividad militar para América del Sur han significado una mayor presencia de nuevos actores en las relaciones militares del continente. ${ }^{3}$ Ello ha dado como resultado una matriz de relaciones mucho más tupida que en décadas pasadas. En los últimos veinte años, desde fines de los años sesenta hasta la actualidad, han proliferado nuevos actores militares extrarregionales todos los cuales han convertido a la región ya sea en una ampliación de su mercado internacional (incluidas las armas), o en una extensión del espacio de competencia político-militar (incluido un cierto número de contingente). Este ciclo de ampliación de los vínculos militares internacionales del continente se ha visto acompañado por el incremento de la cooperación e integración económica entre países de diverso signo político-ideológico.

Sin embargo, paralelo a este proceso de diversificación de las relaciones militares internacionales de la región, América del Sur ha visto aumentar el control politico-militar de las superpotencias sobre las periferias en un marco de crisis y recesión global. El proteccionismo y el repliegue de cada país del norte sobre sf mismo llevarfa, según algunos, a una mayor articulación de las relaciones de poder mundial en torno a las superpotencias, o, según otros, a una recomposición estratégica global ${ }^{4}$. Sin embargo, en ninguno de estos dos escenarios parecería que América del Sur adquiriría nuevos márgenes de maniobra en el plano político y militar.

América Latina: Evolución y perspectivas", en: Gustavo Lagos, (ed,), Las ReLaciones entre América Latina, Estados Unidos y Europa Occidental. Editorial Universitaria. Santiago, 1979.

"Sobre estas nuevas vinculaciones, ver: Isaac Caro, "Relaciones militares de América Latina y el Caribe con Israel y el mundo árabe", Documento de Trabajo No 267, FLACSo-Santiago, octubre 1985; "Relaciones militares đe América Latina y el Garibe con Europa Occidental", Documento de Trabajo No 279, FLAcsoSantiago, enero 1986; "Relaciones militares de América Latina y el Caribe con Estados Unidos y Canadá", Documento de Trabajo No 291, abril 1986.

"Ver, José Agustín Silva Michelena, "Sobre la limitación de transferencia de armas. Notas para la reflexión". Ponencia presentada al seminario "Paz, Desarme y Desarrollo", organizado por RIAL, con el auspicio del PNUD y el patrocinio de FLACso, en Santiago, Chile, 26-27 de mayo de 1986. Cuando nos referimos al papel estratégico estamos hablando de la ubicación y función en la estructura de relaciones militares internacionales. 


\section{El nuevo papel estratégico de América del Sur}

Esta nueva realidad de los años ochenta permite preguntarse si en estas condiciones es o no viable una inserción estratégico-militar de América del Sur, la que se habría posibilitado de continuar las anteriormente señaladas tendencias de corto plazo. Gomo antecedentes de una posibilidad efectiva en este sentido se ha argumentado la erosión de los términos comunes de la defensa hemisférica y del sistema militar panamericanos, y la continuación de la tendencia al aislamiento y la marginalidad de América Latina en el marco del conflicto Este-Oeste. El escaso peso relativo de América del Sur en la transformación de las relaciones internacionales no sería sino producto de su marginalidad estratégica, la que se haría irrelevante en los asuntos internacionales. En consecuencia, no sólo habría existido en los años setenta la necesidad sino que también la oportunidad para intentar jugar un papel estratégico más autónomo. Esta esperanza de independencia estratégica -en el caso que hubiera existido- quedó seriamente dañada, después del conflicto de Malvinas.

Gon todo, pareciera que las opciones que se debatieron en ese período no dieron cuenta de manera adecuada de los verdaderós $y$. reales términos en los cuales se debería haber planteado el tema de la inserción estratégico-militar de América del Sur en el mundo moderno. La polaridad entre marginalidad o protagonismo estratégico-militar se mostró como falsa, en la medida que tal definición, mantenía a la región al interior de los términos tradicionales y.ar, caicos de la defensa territorial decimonónica. En un mundo que ha sufrido transformaciones de significación, la persistencia en defini. ciones obsoletas no pudo sino llevar a mayores frustraciones.

El elemento característico de las posiciones más tradicionales respecto del rol estratégico-militar de América del Sur ha sido el mito de una posición estratégica de América Latina como retaguardia de Occidente controlando sea líneas de comunicación. marítimas, sea espacios de contención. Estas nociones correspondientes al primer período de la guerra fría, momento en el cual la región podría haber jugado este papel, quedaron primeramente obsoletas en la medida que en los sesenta ningún área del globo quedó fuera del alcance del poder destructivo de las superpotencias, lo cual fue profundizado en los setenta y ochenta por su excentricidad en materias técnico-estratégicas. En la guerra moderna; con énfasis básicamenté en lo estratégico-nuclear, América del 'Sur no tíene ningún' papel significativo que jugar. Esta realidad quedó claramente establecida por el general George B. Brown, jefe del estado mayor conjunto de las fuerzas armadas estadounidenses, cuando indicó que "la actual

'Tohn Child, Unequal Alliance: The Inter-American Military System, 19381978. Westview Press, Boulder, 1980, p. 233.

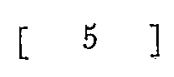


habilidad di: las fuerzas armadas latinoamericanas para la defensa contra una moderna fuerza externa, o para participar en la defensa del hemisferio, es mínima"6. Podríamos sumar una nueva fuente de obsolescencia de las concepciones estratégicas en América del Sur, tal es la crisis de los roles militares referidos exclusivamente a las recién mencionadas posiciones geográficas. Frente a la ampliación de las dimensiones del espacio político-militar moderno que incluye $y$ valora tanto o más que lo territorial al espacio de las comunicaciones $O$ al fondo marino, la visión tradicional de la defensa nacional $y$ hemisférica se ha visto profundamente cuestionada.

La discusión sobre el rol estratégico de América del Sur supone cambiar de óptica, pasando del privilegio otorgado a las posiciones geográficas al énfasis en las condiciones en las cuales América del Sur puede desempeñar un papel como actor, no como territorio, en materias estratégicas. Así, esta nueva concepción no estaría referida necesaria y exclusivamente a la estrecha protección de posesiones terxitoriales. El actor nacional-estatal se desprendería así de su territorialidad para adoptar las formas contemporáneas de un accionar en espacios de diversa naturaleza, comunicativos, comerciales, energéticos, o financieros.

Intentar desarrollar nuevos roles estratégicos en un mundo con

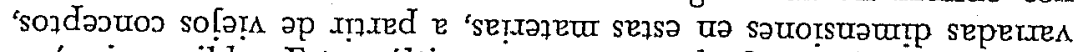
sería imposible. Estos últimos, pocos resultados podrfan exhibir, toda vez que una posición común en estas materias se vería imposibilitada por grandes diferencias geopolíticas, vistas éstas desde un punto de vista estrictamente territorial.

Esta nueva visión de los asuntos estratégico-militares se puede comprender mejor si comparamos las diferentes políticas que surgen de la forma como se han enfrentado y se deberían enfrentar las diversas dimensiones del fenómeno de la seguridad.

El enfoque tradicional de la seguridad interna, la defensa territorial, la seguridad hemisférica y el conflicto entre las superpotencias ha llevado a crecientes ritmos de militarización en el continente. En condiciones de ciclos cortos de autonomía relativa en el campo militar, esta tendencia se ha fortalecido.

En consecuencia, una nueva definición de estas dimensiones debería disminuir y revertir el ritmo de la militarización y, al mismo tiempo, tener respuestas autocentradas que no reproduzcan el fenómeno de la militarización a otro nivel.

El paso de una visión. tradicional a una moderna supone redefinir tanto el objetivo estratégico de América del Sur, como la forma de enfrentarlo, de acuerdo a las nuevas realidades internacionales,

Ver: Department of Defense, Joint Chiefs of Staff, United States Military posture for 1979, Washington, D. C., 1978. 
Augusto Varas / De la competencia a la cooperación militar en América...

Cuadro 1

POLITIGAS DE SEGURIDAD

Concepciones de Seguridad

Dimensiones

Tradicional Moderna

\begin{tabular}{lll}
\hline Interna & $\begin{array}{l}\text { Represión } \\
\text { militar }\end{array}$ & $\begin{array}{l}\text { Acción } \\
\text { policial }\end{array}$ \\
Interestatal & $\begin{array}{l}\text { Militarización } \\
\text { (personal y armas) }\end{array}$ & $\begin{array}{l}\text { Medidas de confianza } \\
\text { mutua }\end{array}$ \\
Regional & Equilibrio & Cooperación \\
& millitar & \\
Global & Inserción en con licto & Intereses \\
& Este/Oeste & propios
\end{tabular}

desarrollando conceptos modernos, diferentes de la represión interna, el conflicto interestatal, el equilibrio militar y la defensa de Occidente.

La definición tradicional de las distintas dimensiones de la seguridad y la defensa -nacional y regional- ha permanecido por décadas en la concepción de las élites dirigentes de América Latina, tanto civiles como militares, sin que las nuevas realidades estratégicas las hayan modificado. Estas concepciones no posibilitan una definición nueva, autocentrada, no heterónoma de la defensa nacional y de la proyección estratégica de América del Sur. En la actualidad el interés estratégico de la región es mucho más amplio que sus intereses militares. Lo militar en sí mismo carecerfa de especificidad si no es en función de la defensa de intereses nacionales, en este caso continentales. La concepción oligárquica tradicional de concebir la defensa territorial y hemisférica confundida irremediablemente al perimetro territorial, privilegiando las funciones policiales de las fuerzas armadas, ha significado un deterioro de la función castrense y un debilitamiento de los términos comunes de las relaciones militares regionales.

En la actualidad, la definión de un rol estratégico moderno para América del Sur supone redimensionar la función policial circunscribiéndola a las conductas penadas por la ley, más que a las

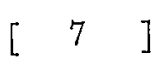


ideologlas, descansando en las instituciones policiales más que en las militares. Igualmente, en materias de defensa interestatal se trataría de lograr niveles superiores de cooperación militar, proyectados a nivel regional, de manera de concebir un interés estratégico común para la región, funcional a sus intereses nacionales actuales. En el campo de la seguridad global se trataría más que de neutralidad y exclusión del conflicto global, un autocentramiento en torno a los intereses regionales. Más que definirse en función de intereses extracontinentales, la región debería concentrar sus esfuerzos en una definición de su papel estratégico en términos positivos. No por referencia a otros intereses, sino en función de los suyos propios. De esta forma, la cooperación militar se podrá incorporar como una de las formas de protección de los intereses comunes de la región sudamericana.

Un nuevo sistema de seguridad regional, propio, producto de una resolución endógena, que no trate de lograr un papel protagónico de América del Sur en materias estratégicas, sino una cooperación regional, protegiendo y al mismo tiempo desarrollando intereses comunes de largo plazo en el plano de la defensa, es una necesidad actual.

La existencia de términos militares comunes posibles para América del Sur han sido develados por la actual crisis económico-social. En medio de una gran diversidad de problemas existe un denominador común, tal es. la necesidad de disminuir la vulnerabilidad externa de la región. Esta tiene a su vez dos fuente fundamentales. Por una parte. la reproducción de la pobreza es un álgido problema de seguridad, puesto que mantiene atadas a las instituciones armadas a concepciones arcaicas que distorsionan la función militar al concentrarlas en materias represivas en lo interno. El peso ideo-. lógico y financiero de las concepciones tradicionales les impide en-. contrar otras fórmulas de defensa nacional que no rea el equilibrio militar regional, lo que a su vez reproduce el ciclo nacional de la pobreza con sus secuelas de mayor carga de la función represiva en las instituciones de la defensa y una nueva y mayor necesidad de equilibrio militar regional. Por otra parte, América del Sur se ha hecho más vulnerable militarmente, en la medida que conflictos desarrollados en otras. áreas tienden a encontrar en la región y en su perímetro náutico y espacial, una extensión del campo de lucha. En las actuales icondiciones de unas fuerzas armadas policializadas, poco.o nada pueden hacer para evitar esta nueva forma de subordinación y sometimiento. Por estas razones, la necesidad de limitar los gastos militares y repensar la seguridad regional son los actuales y modernos denominadores comunes para la región. Éstos son posibles y existentes. El disminuir la vulnerabilidad militar y económica e impedir 'la reproducción de la pobreza, supone nuevas for-' 
mas de cooperación militar en el campo de la seguridad regional. La definición de un nuevo sistema de seguridad ordenaría la polftica de defensa y priorizaría la asignación de recursos y el control del gasto militar, el que hasta el momento ha sido relativamente incoherente y desordenado.

Nuevos objetivos estratégicos autocentrados, tales como el control del hinterland, el espacio y el mar, son tareas imposibles de ser llevadas a cabo por cada país sudamericano en forma aislada. De aquí surge la fuerte necesidad de integración y colaboración para proteger la propia soberanía. La proyección del poder soberano del estado ya no se agota en lo territorial, puesto que se han abierto nuevas dimensiones estratégicas que exigen nuevas respuestas. En la medida que esta proyección del poder soberano requiere de la cooperación regional -condición para ser autónoma y soberana, no subordinada como en el pasado- se encuentra que la cooperación militar sudamericana es en la actualidad la forma más moderna y menos onerosa de afirmacjón de la soberanía nacional.

La Iniciativa de Defensa Estratégica, el plan EUREKA o las res. puestas que la uRss dará a estos desafíos, sólo para mencionar algunos ejemplos, tendrán como efecto una mayor subordinación económica, política y militar de la región latinoamericana respecto de los países del norte. El control sobre el Tercer Mundo impondrá crecientes límites a la soberanía de sus estados, de aquí que la cooperación regional producto de la redefinición del papel estratégico de América del Sur y del encuentro de nuevos conceptos para ello, es una necesidad urgente.

Igualmente, la cooperación civil en los campos científico-tecnológicos para modernizar la defensa colectiva de América del Sur es una opción abierta. Es necesario, sin embargo, prevenir la emergencia de tendencias neo-militaristas a partir de afirmar la necesidad de la cooperación militar regional. Existen experiencias nacionales, en las cuales la modernización, tanto industrial como militar,' ha implicado una nueva militarización, ahora utilizando nuevas tecnologfas y en función de la democratización. En la medida que las fuerzas armadas no desvirtúan sus funciones castrenses en materias policiales, se les ha orientado hacia la modernización e industrialización militar. Sin embargo, la cooperación militar que afirmamos como necesaria y posible debería evitar tales situaciones. Primeramente, porque la modernización de la industria militar lanza al país respectivo a un mercado internacional ya saturado de productos militares. En segundo lugar, porque esta modernización ${ }^{i}$ se hace a costa de recursos fiscales, los que ya no se usan en contingentes voluminosos pero que se utilizan, en investigación, desarrollo y producción de armamento. "Tercero, porque la modernidad en este cámpo está siendo liderada, por la IDE y. EUREKA, de modo que 
una vinculación a ellas implicarf́a reproducir la alienación estratégica tradicional, descentrando a América del Sur de sus propios intereses estratégicos, e impidiéndole encontrar los nuevos.

Una política de desarrollo a largo plazo debería servir para desarrollar la nueva capacidad militar regional cuyo objetivo sería evitar sus vulnerabilidades económicas y militares. La modernización se pondría al servicio de la seguridad regional. No como en el pasado, cuando la seguridad y los militares se vaciaban de sus funciones castrenses en aras de objetivos civiles desarrollistas, capacitando mano de obra o produciendo herramientas. El resultado de esta política fue la invasión castrense del conjunto del estado. En una nueva concepción de seguridad el conjunto del estado latinnamericano debería ser competente para llenar y ocupar un nuevo Jugar estratégico, independiente, no hegemónico, en el mundo moderno. Integrando, junto a otras instituciones y estructuras, a las propias fuerzas armadas, evitando convertirlas en el eje de coordinación y coherencia del estado. La ausencia de un sistema de seguridad latinoamericano autocentrado concebido y administrado por cl conjunto del estado, ha sido el producto de un gran vacío en las concepciones estratégicas de los civiles. Cuando esto sucede, tal vacío es llenado por las FF. AA. en forma subsidiaria y, muchas veces, de manera inadecuada.

Un nuevo sistema de seguridad debe ser visto como el inicio de una secuencia que implicaría ir logrando niveles progresivos de paz. Disminuyendo las tensiones internas, creando medidas de confianza mutua, logrando integraciones militares parciales, importando y produciendo menos armas y generando más desarrollo, será posible avanzar hacia proyectos más globales de integración y desarme.

\section{Las potencias extrarregionales}

Este nuevo sistema de seguridad deberfa modernizar las relaciones en el plano estratégico con potencia extra-regionales. Afirmamos la modernización como elemento articulador de esta redefinición, puesto que estimamos inconveniente fundamentar un sistema de seguridad regional exclusivamente frente a peligros extra-regionales. Aquf también encontramos un nuevo mito, el de la fortaleza sitiada, esto es la solución de conflictos vía la cohesión interna frente a una amenaza externa. Este mito tiene serias limitaciones. Por una parte, la integración estratégica regional no asegura por sí sola la eliminación de la confrontación entre miembros del sistema. El caso más gxáfico es el del conflicto entre Grecia y Turquía en el marco de la OTAN; o de la guerra entre El Salvador y Honduras en el contexto del TIAR. Esta integración estratégica es condición necesaria, mas no suficiente para inhibir conflictos intra-regionales.

La redefinición del papel estratégico de América del Sur debería 
poner mayor-énfasis en un sistema de seguridad que disminuya las posibilidades de conflicto entre páses del área en forma gradual, evitando opciones radicales, acelerando la cooperación militar y nuclear pacífica, evitando la polarización militar regional en torno a centros de poder o alianzas intra-regionales, $y$ atenuando las percepciones de amenaza a la seguridad de cada uno de los países sudamericanos.

La opción estratégica de América del Sur debería ser una afirmación de su propia personalidad militar, no-competitiva con potencias militares extra-regionales, evitando integrar a América del Sur en el conflicto Este-Oeste, sea a través de la alineación, sea a través de una fuerza de disuasión propia. Esta opción de independencia y autocentramiento estratégico incluso podría ser funcional a los intereses estratégicos de EE. UU. $y$, con su apoyo pero bajo control sudamericano, evitando instrumentalizaciones, constituirse en un actor estratégico con deliberada afirmación de individualidad. Una posición de este tipo sería funcional a los Estados Unidos, porque influiría en la posición global de éstos al no tener que desviar atención y recursos en forma permanente a un flanco no siempre confiable.

\section{Cuadro 2}

TIPOS DE GOOPERAGION MILITAR ITEMISFERICA

América

Intra Sudamericana

Extra Sudamericana

Percepción $\quad$ Centroamérica $\quad$ Malvinas
de amenaza

Estados

Unidos

$\begin{array}{lll}\begin{array}{l}\text { Percepción de } \\ \text { colaboración }\end{array} & \begin{array}{l}\text { Ghile- } \\ \text { Argentina }\end{array} & \text { Crisis de los misiles } \\ & \text { Cuba } 1962\end{array}$

Tal como se puede apreciar en el esquema anterior, la profundidad y extensión de las crisis militares en la región latinoamericana han sido producto de la percepción de los Estados Unidos de una amenaza a sus intereses estratégicos, cualquiera sea la naturaleza de ellos. Por el contrario, cuando esta percepción es de colaboración hemisférica, el papel de éstos es positivo, ayudando a disminuir los 
focos de tensión continental. Por estas razones, nos atrevemos a afirmar que un sistema de seguridad regional, tal como el que se ha delineado debería considerar la relación con los Estados Unidos como uno de los elementos de mayor importancia para la factibilidad de su desarrollo.

Esta nueva relación con la potencia hegemónica en el área supondría generar una redefinición estratégica de las potencias militares de la zona, éstas son Argentina y Brasil. Esta decisión implicaría aumentar la confianza entre estos países para cooperar en materias convencionales, tal como ya lo están haciendo, y profundizarlas en materias nucleares como se lo han propuesto ${ }^{7}$. La necesidad de concertación argentino-brasilera evitaría la competencia, confrontación, polarizaciones y sistemas de alianzas regionales. El integrar a Brasil a la región recuperando su actual presencia en las cuencas del Plata, Amazonas y Orinoco, proyectaría al conjunto de Sudamérica hacia formas deliberadas y pacíficas de colaboración. A partir de este eje dinámico Argentina-Brasil, América del Sur podría autocentrarse formulando las bases para elaborar sus propios intereses estratégicos.

Los nuevos temas estratégicos surgen necesariamente de una afirmación diferente de los intereses regionales. Así, sería posible pensar en torno a una Agencia Espacial Latinoamericana, o en una OPANAL como fórmula de desarrollo de un sistema propio de salvaguardias para el programa nuclear regional, o en una industria militar regional integrada con capacidad de aprovechar lo existente en función de desarrollos civiles.

\section{Desarme y desarrollo}

Se ha visto que en la actualidad no hay acuerdos significativos váIidos de limitación de armamentos en la región. La principal razón por la cual se ha limitado el gasto militar y la importación de armas en los últimos años ha sido producto de la crisis económica, Esta situación muestra que el problema técnico es superable, pero que los obstáculos a la limitación de armamento son básicamente políticos.

De acuerdo a las consideraciones realizadas sobre el nuevo rol estratégico de América del Sur, será posible limitar y reorientar el gasto militar de acuerdo a esta concepción y del elemento que prima al interior de ella, este es el interés nacional regional.

Considerando que las conversaciones sobre el desarme y control

TLa cooperación nuclear argentino-brasilera se da en el campo de las aplicaciones civiles, no en aquellas áreas susceptibles de ser utilizadas en la perspectiva de la seguridad, lo cual mantiene un margen de competencia $y$ desconfianza mutuạ. 
de armamentos tienen como primer objetivo el evitar la guerra, asimismo, el control, limitación, reducción o eliminación del gasto en armamentos para posibilitar el desarrollo, pueden ser concebidos como pasos dentro de una progresión más amplia. Teniendo objetivos crecientemente envolventes en un mismo y largo proceso. De la misma forma, considerando que este itinerario en definitiva queda supeditado por quien está por conceder lo menos, es necesario imaginarlo y aceptarlo como una secuencia de iniciativas diversas, pero que en el largo plazo se articulan unas a otras.

Cuadro 8

ALTERNATIVAS DE POLITICAS DE DESARME

\begin{tabular}{lcccc}
\hline Pollticas & Presupuesto & Gasto & Importación & Industria \\
\hline Controlar & $(1)$ & 2 & 3 & 4 \\
Limitar & 5 & $6^{*}$ & $7 *$ & 8 \\
Reducir & 9 & 10 & 11 & 12 \\
Eliminar & $(16)$ & 15 & 14 & 13 \\
\hline
\end{tabular}

"Primeros pasos.

Así, los procesos de desarme muestran una amplia gama de posibilidades, para cada una de las cuales hay que desarrollar instrumentos adecuados. Esta, parte de las iniciativas de control de presupuestos y puede llegar hasta la eliminación de cierto tipo de gastos. Existen dos elementos comunes en todas y cada una de estas alternativas de desarme, tales son, en primer lugar, la transformación de la percepción de amenaza en percepción de colaboración y seguridad, y, la segunda, la necesidad de adecuar el dispositivo bélico de acuerdo a las nuevas percepciones. De esta forma estamos afirmando que los dispositivos militares no tienen necesariamente que desarrollarse en un espiral ascendente, sino que éstos pueden variar de acuerdo al sistema de percepciones nacionales sobre seguridad y amenaza en la región ${ }^{8}$.

sobre este punto ver los trabajos presentados a la "Reunión de Expertos sobre las Percepciones de Amenaza y Seguridad", organizada por unesco en Parfs, del 2 al 5 de noviembre de 1982. Respecto a América Latina, ver: Augusto Varas, "Climat de Securité et Perception de la Menace dans les Pays' D'Ámérique Latine: Tendances et Propositions en Vue de Recherches Futures", unssco, ss-32/ CONF. $614 / 10,25$ de noviembre 1982. 
El objetivo del desarme puede pasar por una amplia variedad de iniciativas de limitación del gasto en defensa, manteniendo al mismo tiempo un balance militar regional. En la medida que el control o reducción de algunos aspectos del gasto militar requieren una profundización particular en esos temas, es necesario avanzar en un análisis cualitativo de 'as propuestas de desarme, estudiando el tipo de armamentos que se propone reducir o limitar, sobre todo cuando se incorpora en las negociaciones de desarme la idea de contener la importación y producción de armamentos. Este enfoque cualitativo abre el paso al desarrollo de conceptos que ponen énfasis en una "defensa defensiva", la que con el paso del tiempo permitirfa reducir o eliminar ciertos rubros que abultan los presupuestos de defensa. Por estas razones, sería prudente avanzar hacia un estudio más pormenorizado del balance estratégico-militar intrarregional. Este procedimiento podría ir desde la asignación de puntajes a los diversos tipos de armas, hasta la limitación de cierto tipo de gastos en relación al PNB, quedando cada país en libertad de asignar sus recursos en otros rubros.

Un apoyo eficaz para la limitación de armamento es el desarrollo de medidas de confianza mutua. Las visitas recíprocas de jefes militares (que requieren una solución de continuidad dada la rotación individual en los cargos), es un elemento primario de importancia. De la misma forma, el intercambio de cuotas de oficiales en escuelas profesionales, el uso del arreglo militar directo en situaciones críticas, el intercambio de información, el distanciamiento de tropas de las fronteras, las maniobras conjuntas sin Estados Unidos ${ }^{\theta}$ son ejemplos expresivos de lo que estamos señalando. En este marco se ubica, por ejemplo, la posibilidad de reproducir con otros países las conversaciones sostenidas últimamente entre Chile y Perú. Es necesario reiterar que los procesos de integración económica, cultural y social, son en sí mismos una excelente forma de generar confianza mutua entre los estados de la región.

Un aspecto altamente sensible para las posibilidades de avance en materias de desarme o limitación de armamentos, es el problema del control y verificación de los acuerdos logrados. Indudablemente, acuerdos de este tipo implicarían ciertas limitaciones a la soberanía de cada estado individual. Sin embargo, a este respecto caben dos consideraciones. La primera dice relación con la necesidad de mantener permanentemente a la vista el papel estratégico conjunto de América del Sur. De aquí surge la necesidad de desarrollar los medios técnicos eficaces para un sistema regional de verificación y control de la acción de potencias extra-regionales en el área, las que limitan la soberanía compartida. La segunda, nos remite a la

'Tal es el caso de las llevadas a cabo por Argentina, Brasil y Uruguay para la protección de convoyes. 
necesidad de desarrollar los medios técnicos para sostener este sistema regional de verificación y control. De esta manera en la medida que América del Sur esté desarrollando los términos y los medios colectivos para asegurar su soberanía regional, al mismo tiempo estos medios y nuevos conceptos podrán ser utilizados para verificar y controlar la recta aplicación de los acuerdos intra-regionales. Como ejemplo de esto podríamos mencionar la posibilidad de controlar y verificar, vía satélite, el estacionamiento de tropas cercanas a las fronteras. Así, la cooperación científica-tecnologica para desarrollar medidas adecuadas de verificación y control, propias y soberanas, sin pagar costos políticos de subordinación, es una opción realista y viable en el mediano plazo.

Finalmente, cabe señalar que los recursos liberados por una eventual limitación de armamentos no tienen una asignación mecásica o automática hacia planes de desarrollo. La reducción del gastc militar, en unuchos países sudamericanos, se consume en pagar la deuda externa, no en programas de desarrollo. El desarme y la limitación de armamentos está íntimamente relacionado con la necesjdad de producir desarrollo como única alternativa de disminuir la vulnerabilidad de la región. Detener $y$ eliminar el ćrculo vicioso de la reproducción de la pobreza es en sl mismo un objetivo de seguridad nacional y regional. En consecuencia, existe la necesidad de asignar estos recursos a un tipo nuevo y diferente de desarrollo.

Si es difícil lograr medidas de confianza mutua o de limitación de armamento, más aún sería poner de acuerdo a las fuerzas políticas lócales en torno a qué tipo de desarrollo sería necesario apoyar con los recursos liberados del gasto en defensa. Sin embargo, existen tres tipos de asignaciones que, cualquiera sea la opinión u opciós sobre las alternativas de desarrollo se deberían considerar, tales son los gastos en salud, educación y vivienda. Estas son asignaciones comunes a cualquier tipo de desarrollo endógeno alternativo.

\section{El mercado de armamentos}

La industria militar sudamericana es parte de la política exterior de sus respectivos estados, los que la utilizan como un mecanismo para copar ciertos espacios estratégico-políticos que las grandes potencias no pueden llenar. Sin embargo, tal como se ha desarrollado, la industria militar regional bloquea las iniciativas de limitación de armamentos ${ }^{10}$. De igual forma, la industria militar regional no beneficia al desarrollo global nacional, sino a empresas individuales, desarrollándose fundamentalmente por razones de poder nacional vía subsidios estatales. Incluso existen dudas sobre su eventual efi-

10Y afecta la propia logfstica nacional, clave para conflictos de larga duración.

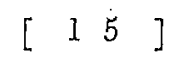


ciencia y aporte al crecimiento local, en la medida que falta información confiable sobre sus beneficios efectivos.

Por las razones anteriores, parece altamente conveniente integrar las industrias existentes a nivel regional con un triple política. Por una parte, hacerlas más eficientes aliviando la carga financiera sobre los presupuestos nacionales. Segundo, generando polfticas de confianza mutua entre los estados de la región. $Y$, tercero, proyectándolas hacia volúmenes crecientes de producción para uso civil. Esta política permitiría una conversión de hecho de la industria militar regional dándole una polivalencia deliberada, sin crear nuevos desequilibrios militares regionales.

En la medida que se han reducido las tasas de importación de armas por parte de América del Sur, ha aumentado la capacidad de negociación de los compradores. Igualmente, es necesario reconocer que la iniciativa de integración industrial militar no provendrá de Ios proveedores. Así, la actual capacidad de producción y exportación de armas de América Latina, mediante una voluntad política para hacerlo, podría transformarse en un elemento positivo apto de ser utilizado en función de la distensión y la limitación de armamentos, así como para lograr una mayor capacidad de negociación y control sobre los proveedores de tecnología militar.

Dada la creciente transnacionalización de la industria de armas, de hecho a América del Sur se le dificulta ejercer su soberanía en materias de producción y aprovisionamiento. La integración industrial militar - apoyada por un sistema de cooperación científica-tecnológica regional- también puede ser vista en la misma línea de desarrollo de un interés auto-centrado que le da forma a la idea del nuevo papel estratégico de América del Sur. Considerando que el costo de la industria militar para pafses pequeños es mayor que para países más grandes, el beneficio de una integración alcanzaria a todos los estados regionales, logrando de esta forma un amplio consenso potencial. Finalmente, cabe señalar que el papel del estado en este proceso es central, debiendo quedar bajo dirección y control civil el conjunto del proceso en cuestión.

\section{Los desarrollos en el campo nuclear}

La combinación de los avances en el campo de la energfa nuclear en América del Sur, con las pruebas en el Pacífico Sur y la presencia de armamento nuclear en el Atlántico Sur, están generando una cierta crisis de la confianza mutua en estas materias de la región.

Uno de los argumentos más serios para no aceptar plenamente el Tratado de Tlatelolco, es la discriminación contenida en el sistema de verificación y control de éste, el que proviene del tratado de NoProliferación Nuclear, favoreciendo a las potencias nucleares en 
desmedro de Ios países del Tercer Mundo. La Organización Internacional de Energia Atómica no ha respondido a las propuestas alternativas para adecuar estas cláusulas a la independencia sudamericana. De la m:sma forma, el Club de Londres regula la política discriminatoria frente a este mismo grupo de países. Dadas las reservas de los principales estados sudamericanos frente al tratado de Tlatelolco, y considerando la creciente vulnerabilidad nuclear del continente en su conjunto, difícilmente se podría visualizar una efectiva desnuclearización de la región.

Se ha afirmado que algunos de los programas nucleares en el área no estarían motivados por una necesidad energética, sino más bien por razones de seguridad y prestigio militar. Igualmente, otros programas mantienen cierta ambigüedad respecto a su posible uso, sea como forma de desarrollar un arma nuclear o de aplicar esta exiergía a barcos o submarinos.

Por su parte, la cooperación nuclear intra-sudamericana ha dejado claramente afuera aquellas materias que dicen relación con el uso militar potencial del ciclo nuclear. De esta forma, surge la legitima aprensión por la existencia de una opción indirecta por el desarrollo bélico nuclear, perfeccionando el ciclo nuclear pacifico, derivándolo potencialmente hacia fines de seguridad.

En la medida que no hay forma de controlar la difusión nuclear en la región bajo las actuales condiciones, es necesario concebir al tratado de Tlatelolco como un acuerdo en desarrollo capaz de perfeccionarse y adaptarse a las nuevas realidades.

Una de estas nuevas realidades son los crecientes altos costos de lá energía nuclear en el contexto de la baja de los precios del petróleo, otra es la crisis de seguridad por la que pasan estos programas. Tales son los casos de las catástrofes de Three Miles Island y Chernobyl. Las medidas para mejorar la seguridad de los reactores implicarían mayores costos aún, por lo cual la solución de aumentar su tamaño y capacidad de producción de energía sobrepasaría de lejos las necesidades energéticas de la región.

De acuerdo a esta situación existe la necesidad de revalorizar los programas nucleares regionales y generar salvaguardias que conduzcan a una efectiva confianza mutua. Un plan de desarrollo en esta línea indicaría la conveniencia de una ratificación del tratado de Tlatelolco sobre la base de un nuevo sistema de salvaguardias generadas en torno a OPANAL. Igualmente, se requiere una posición política clara del conjunto de países con programas nucleares, cualquiera sea su nivel de desarrollo, sobre la necesidad y conveniencia de la desnuclearización del Pacífico y Atlántico Sur. Estas medidas, junto a posiciones comunes frente a la actual status de la Antártica, aumentarfa sensiblemente la posición de negociación colectiva de la región en materias nucleares. La vulnerabilidad de América 
del Sur en estos campos, difícilmente se logrará a través de úna disuasión nuclear propia, por lo cual su única opción realista es el elevar su nivel de cooperación científico-tecnológica regional para evitar tanto sus propios desarrollos, como el riesgo nuclear extracontinental.

\section{El papel de la opinión pública}

Hemos visto la importancia que tiene para asegurar la paz y el desarme la percepción de seguridad o amenaza existente al interior de las élites militares y civiles. De aquí surge un primer objetivo de política previo a cualquier iniciativa de limitación de armamentos, tal es el cambio y transformación de estas percepciones.

Una primera forma de iniciar este proceso es la producción y difusión más amplia de la información sobre el equilibrio militar regional. Así, el conjunto de la opinión pública sudamericana, tal como se pronuncia sobre las condiciones económicas nacionales, podría tomar un papel más activo en las decisiones y acciones realizazas por los respectivos gobiernos en el plano de los desarrollos de seguridad.

AI respecto existe la urgencia de cumplir con los compromisos aceptados por los países sudamericanos de informar a las Naciones Unidas de los gastos militares según el esquema aprobado ${ }^{11}$. Hasta el momento casi ningún gobierno de la región informa de acuerdo a esta pauta, única manera de lograr estandarizar la informac'ón y avanzar hacia propuestas realistas. En este campo de problemas, los organismos no-gubernamentales locales tienen una responsabilidad de primer orden frente a sus respectivas audiencias locales.

Una segunda medida necesaria de ser adoptada es la creación de un foro adecuado para debatir estos temas a nivel sudamericano, en el cual se posibilite el encuentro de quienes tienen un papel en la toma de decisiones sobre política exterior y militar, junto a técnicos civiles de la región.

Finalmente, creemos que todos los pasos que se den en materia de conversaciones sobre medidas de confianza mutua llegando hasta el desarme, requieren de una contrapartida técnico-académica apta y solvente para apoyar estos desarrollos desde la perspectiva del interés regional. El natural y legítimo énfasis en los intereses nacionales, debería ser compensado por un sistema de coordinación académica regional que posibilitara la emergencia de nuevos conceptos regionales, en la perspectiva de imaginar un nuevo papel estratégico para América del Sur que proporciene paz y desarrollo a sus pueblos.

دVer: United Nations, R'eduction of Military Budgets, New York, 1981; especialmente pp. 81 a 42 . 